\title{
Sums of Distinct Primes from Congruence Classes Modulo 12
}

\author{
By Robert E. Dressler, Andrzej Mạkowski and Thomas Parker
}

\begin{abstract}
It is shown that every integer greater than $1969,1349,1387,1475$ is a sum of distinct primes of the form $12 k+1,12 k+5,12 k+7,12 k+11$, respectively. Furthermore, these lower bounds are best possible.
\end{abstract}

In [2], A. Mąkowski proved the following:

THEOREM. Every integer greater than 55, 121, 161, 205 is a sum of distinct primes of the form $4 k-1,4 k+1,6 k-1,6 k+1$, respectively. Furthermore, these lower bounds are best possible.

To do this, he used the work of Breusch [1] and the following theorem of H. E. Richert [4].

THEOREM. Let $m_{1}, m_{2}, \cdots$ be an infinite increasing sequence of positive integers such that, for some positive integer $k$, the inequality $m_{i+1} \leqq 2 m_{i}$ holds for all $i>k$. Suppose there exists a nonnegative integer $a$, such that the numbers $a+1, a+2, \cdots$, $a+m_{k+1}$ are all expressible as a sum of distinct members of the set $\left\{m_{1}, m_{2}, \cdots, m_{k}\right\}$. Then, every integer greater than $a$ is expressible as a sum of distinct members of the sequence $m_{1}, m_{2}, \cdots$.

Our purpose is to strengthen Mąkowski's work by using Richert's theorem and the following theorem of Molsen [3].

THEOREM. For $n \geqq 118$, the interval $(n, 4 n / 3)$ contains a prime of each of the forms $12 k+1,12 k+5,12 k+7,12 k+11$.

Our work was done on the IBM 360/50 computer at Kansas State University and we wish to thank Mr. Gary Schmidt for his help in the programming. The program was written in FORTRAN and the computer time was 12.42 minutes. So much time was used because we wanted more information than we actually needed to prove the theorem. For example, we obtained information about the multiplicities of representation.

We now give our result.

THEOREM. Every integer greater than 1969, 1349, 1387, 1475 is a sum of distinct primes of the form $12 k+1,12 k+5,12 k+7,12 k+11$, respectively. Furthermore, these lower bounds are best possible.

Proof. In the case of $12 k+1$, put $a=1969, m_{k}=2029$, and $m_{k+1}=2053$. In the case of $12 k+5$, put $a=1349, m_{k}=1301$, and $m_{k+1}=1361$. In the case of $12 k+7$, put $a=1387, m_{k}=1327$, and $m_{k+1}=1399$. In the case of $12 k+11$, put $a=1475, m_{k}=1523$, and $m_{k+1}=1559$.

Received June 20, 1973.

AMS (MOS) subject classifications (1970). Primary $10 \mathrm{~J} 15$.

Key words and phrases. Primes, congruence classes modulo 12.

Copyright $\odot 1974$, American Mathematical Society 
Department of Mathematics

Kansas State University

Manhattan, Kansas 66502

Institute of Mathematics

University of Warsaw

Warsaw, Poland

Department of Mathematics

Kansas State University

Manhattan, Kansas 66502

1. R. Breusch, "Zur Verallgemeinerung des Bertrandschen Postulates, dass zwischen $x$ und $2 x$ stets Primzahlen liegen," Math. Z., v. 34, 1932, pp. 505-526.

2. A. MArowski, "Partitions into unequal primes," Bull. Acad. Polon. Sci. Sér. Sci. Math. Astronom. Phys., v. 8, 1960, pp. 125-126. (Russian) MR 22 \#7991.

3. K. MoLsEN, "Zur Verallgemeinerung des Bertrandschen Postulates," Deutsche Math., v. 6, 1941, pp. 248-256. MR 8, 197.

4. H. E. RICHERT, "Uber Zerlegungen in paarweise verschiedene Zahlen," Nordisk Mat. Tidskr., v. 31, 1949, pp. 120-122, MR 11, 646. 